\title{
Aula invertida en pandemia: Análisis de una experiencia disruptiva desde la mirada de los representantes
}

Flipped classroom in a pandemic: Analysis of a disruptive experience from the perspective of the representatives

\author{
Angélica María Sánchez-Santander \\ Angelica.sanchez03@est.ucacue.edu.ec \\ Universidad Católica de Cuenca, El Tambo \\ Ecuador \\ https://orcid.org/0000-0002-8877-243X \\ Nancy Marcela Cárdenas-Cordero \\ ncardenasc@ucacue.edu.ec \\ Universidad Católica de Cuenca, Azogues \\ Ecuador \\ https://orcid.org/0000-0002-6250-6504
}

Recepción: 15 de marzo 2021

Revisado: 15 de mayo 2021

Aprobación: 15 de junio 2021

Publicación: 01 de julio 2021 


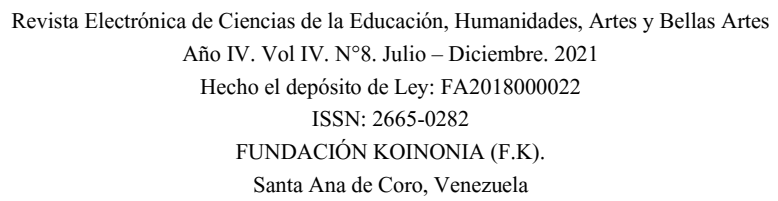

\title{
RESUMEN
}

En la actualidad nos encontramos atravesando épocas de grandes cambios, tal es el caso del sector educativo que a raíz de la pandemia generada por la Covid-19 ha orillado a los docentes a utilizar recursos tecnológicos y metodologías activas en el afán de fortalecer el proceso de enseñanza aprendizaje. El objetivo de la presente investigación es Analizar cómo la aplicación del aula invertida puede aportar al aprendizaje de estudiantes desde la percepción de los representantes o padres de familia. De corte cuantitativo, descriptiva con diseño no experimental. El análisis del resultado evidencia que dicha metodología goza de gran popularidad tanto en estudiantes como en representantes ya que se ha comprobado que mejora el rendimiento académico frente a métodos tradicionales. Pero cabe señalar que por sí sola la aplicación del aula invertida no garantiza la mejora del desempeño académico puesto que son varios los factores que influyen en el proceso educativo.

Descriptores: Enseñanza centrada en el rendimiento; enseñanza multimedia; tecnología educacional. (Palabras tomadas del Tesauro UNESCO).

\begin{abstract}
At present we are going through times of great changes, such is the case of the education sector that as a result of the pandemic generated by Covid-19 has forced teachers to use technological resources and active methodologies in an effort to strengthen the process of teaching learning. The objective of this research is to analyze how the application of the flipped classroom can contribute to student learning from the perception of the representatives or parents. Quantitative, descriptive cut with non-experimental design. The analysis of the result shows that this methodology is very popular in both students and representatives since it has been proven that it improves academic performance compared to traditional methods. But it should be noted that by itself the application of the flipped classroom does not guarantee the improvement of academic performance since there are several factors that influence the educational process.
\end{abstract}

Descriptors: Competency based teaching; multimedia instruction; educational technology. (Words taken from the UNESCO Thesaurus). 


\section{INTRODUCCIÓN}

La presente investigación buscar analizar la incidencia que tiene la Flipped Classroom o aula invertida en el proceso de enseñanza -aprendizaje de los estudiantes en todos los niveles de educación. Para esta investigación se analiza los trabajos realizados a nivel mundial se analiza el de (Sánchez-Cruzado, 2017), en la investigación realizada en España en el nivel superior indica que al existir un alto índice de estudiantes que asisten a clases se sienten desconectados en un aula con sobre población estudiantil, y no les interesa continuar dentro de ella, esto es un aspecto negativo para su formación. Surge así la necesidad de que los docentes recurran a la formación y utilización en herramientas digitales, a su aplicación para conseguir motivar al estudiante a continuar en clases.

A nivel regional en México se desarrolló la investigación de (Parra-Acosta et al., 2015), quienes en su investigación determinaron que no existen suficientes estudios en cuanto a la socio-formación de los docentes por mejorar la pedagogía al momento de impartir sus clases. Lo que implica una dificultad esto ha hecho de que los docentes no se encuentren capacitados en metodologías constructivistas, por consiguiente, exista una escasa atención y motivación al momento de asistir a clases.

Ahora se analiza que (Mendoza-Castillo, 2020), en su investigación menciona que, en el nivel de educación primaria en la modalidad presencial no existen los recursos económicos necesarios que garanticen que el $100 \%$ de los estudiantes tengan acceso a las nuevas tecnologías, pues la mitad de la población estudiantil se encuentra sumida en la pobreza, y en estas condiciones es preciso analizar si el utilizar recursos tecnológicos ofrece la solución esperada, por consiguiente se debe tener presente que en la mayoría de países, la situación económica es un factor limitante para conseguir el acceso a una educación virtual de calidad.

Mientras que en el Ecuador se reconoce el trabajo de (Molina-Naranjo et al., 2018), en donde se realiza un estudio sobre los modelos educativos prevalecientes en el país, del cual se concluye que el modelo tradicional todavía está vigente en los niveles educativos, pues aún existen docentes que son el centro del proceso educativo y quienes tienen el 
control en cuanto a la formación de sus estudiantes, convirtiéndolos así a los estudiantes en simples receptores pasivos de información y todo el proceso de enseñanza aprendizaje gira en torno a los maestros o docentes.

En si el problema central es determinar la falta de actualización docente y de cambio de modelos educativos para conseguir la calidad educativa esperada, es decir que los docentes sean capaces de reconocer sus debilidades metodológicas y estén es la disponibilidad de aprender, auto educarse y a adquirir competencias digitales acordes a las exigencias de una sociedad que cambia constantemente, pues nos encontramos frente a nuevos escenarios, disponemos de recursos novedosos que pueden ser adaptados a las necesidades estudiantiles de la actualidad, pero lamentablemente no toda la población estudiantil cuenta dispositivos que le permitan hacer uso de ellos para acceder al conocimiento.

La escasa motivación del estudiantado se ha visto reflejado en el rendimiento académico esta problemática a su vez ha ocasionado deserción estudiantil es por esto que se busca con este proyecto de investigación Analizar si la aplicación del aula invertida puede aportar al aprendizaje de estudiantes desde la percepción de los representantes o padres de familia.

\section{Referencial teórico}

En el siglo XXI son innegables los cambios que todas las organizaciones han atravesado a lo largo de la historia y sobre todo en educación, los cuales han sido motivo de análisis por varios investigadores siendo el caso de (Belfar et al., 2003) en su investigación realizada en Costa Rica menciona que de estos cambios no se libra el sector educativo, pues debido a su enorme responsabilidad en la formación de individuos debe afrontar notables disyuntivas, en su labor con el propósito final de ofertar procesos educativos de excelente nivel. cabe indicar que para conseguir el tan esperado nivel educativo es fundamental el cambio en el accionar del docente. 
El profesorado debería meditar y dejar de lado la educación tradicional pues este modelo pedagógico según (Domínguez et al. 2015), afirman en su investigación que en el modelo tradicional el profesor dicta un tema que si bien trata de persuadir al estudiante a revisarlo con mayor profundidad, no siempre logra su objetivo ya que los alumnos suelen aburrirse con mayor facilidad, siendo verdaderamente difícil lograr un aprendizaje significativo que motive o despierte en el estudiante un verdadero interés por dominar el conocimiento impartido por el profesor. A diferencia de la metodología activa aula invertida o Flipped Classroom en la cual a los estudiantes se les provee de los recursos en los cuales se apoyarán como son libros, vídeos Flipped, podcast, antes de iniciar la clase.

Esto ofrece las herramientas necesarias para que el estudiante profundice en mayor medida en el tema, a la vez que no limita su entorno de aprendizaje a un aula sino se adapta al entorno del estudiante, esto a más de ser fuente en si misma de motivación para el estudiantado, genera un mayor discernimiento y pensamiento crítico, a más de otros valores como responsabilidad, autonomía entre otros. Cabe señalar la importancia de utilizar la tecnología como un soporte para el aprendizaje pues los materiales utilizados en el método tradicional comienzan a ser anticuados o con poca utilización en nuestro nuevo entorno tecnológico.

Es oportuno ahora analizar la investigación realizada en Colombia por (Jensen et al. 2015), en la cual el autor afirma que la falta de interés en las aulas por parte de los estudiantes es cada vez más evidente, pues cualquier estimulo provoca en ellos gran distracción y trunca el proceso de aprendizaje, el mundo tecnológico en el cual estamos inmersos invita al docente a formularnos la pregunta si la manera en la que estamos llevando el conocimiento a nuestros estudiantes es la mejor, o si por el contario somos nosotros como docentes quienes también somos responsables en cierto grado de esta falta de interés.

Alrededor de la temática se analiza el trabajo investigativo realizado en México por (Aguayo-Vergara et al. 2018), el objetivo fue promover el consumo y producción de 
recursos educativos se aplicó una estrategia de aprendizaje dividida en tres momentos en la que se implementó la Flipped Classroom, creación de recursos y evaluación al inicio se aplicó una escala tipo Likert, los resultados revelan, que los estudiantes valoran la posibilidad de contar este tipo de material como videos con el contenido previo inicio de clases. En esta línea de argumentación cabe indicar que se sintieron protagonistas de su conocimiento y percibieron al docente como un facilitador en su proceso de aprendizaje. Finalmente se concluye que la metodología diversifica los momentos del aprendizaje, fortalece los conocimientos previos, y garantiza un proceso de enseñanza aprendizaje centrado plenamente en el estudiante, más del 90 por ciento de los alumnos coinciden en que esta metodología les permitió mejorar su aprendizaje y habilidades para comunicarse, adicional a esto se desarrollaron habilidades como: el pensamiento crítico, la capacidad de resolver problemas y su motivación en clase mejoró notablemente.

El trabajo investigativo se relaciona, con la teoría experiencial de (Granados-López \& García-Zuluaga, 2016), en el cual se buscó determinar si las predominancias de aprendizaje en estudiantes de cuarto grado de una institución educativa, modifican o no su aprendizaje al aplicar en el aula, el modelo de aprendizaje experiencial.

En cuanto a la conceptualización del aula invertida, según (Sosa-Díaz \& Palau-Martín, 2018), determinan que el aula invertida se encuentra a la vanguardia en cuanto a metodologías pedagógicas ya que esta provee al estudiante de un sin número de habilidades que hacen que el proceso de enseñanza aprendizaje sea propicio, como se dijo al principio es preciso hacer un cambio en cuanto a lo que metodología ya que son numerosos los beneficios, que los estudiantes obtienen mediante la aplicación de este enfoque activo durante el desarrollo del proceso formativo en las aulas. Sin embrago no existen suficientes estudios que evidencien las ventajas o desventajas de trabajar con esta metodología.

Indicaremos que según los estudios arrojados y descritos por los mismos estudiantes los beneficios obtenidos son: Adaptación al ritmo del estudiante, los alumnos se sienten más motivados al contar con materiales que pueden utilizar según su nivel de comprensión 
evitando así la frustración estudiantil, repetición de contenidos, interacción social el estudiantado podrá hacer trabajo colaborativo, y a su vez fomenta el aprendizaje autónomo.

Continuaremos con la exploración según la investigación realizada por (Domínguez et al., 2015), manifiestan que la estrategia aula invertida es una alternativa en la que los papeles se invierten, es decir el rol del maestro, es ser un guía, un facilitador un mediador en el proceso de enseñanza aprendizaje, el papel preponderante lo toma el estudiante, como eje central de todo el proceso educativo, a los estudiantes se les presenta materiales como libros, videos, podcast antes de iniciar la clase para que mediante esta actividad se potencie el aprendizaje activo, el pensamiento crítico aquí es de vital importancia la incorporación de la tecnología, por cuanto los métodos tradicionales no satisfacen las necesidades y exigencias actuales.

Conviene sin embargo advertir que en trabajos investigativos realizados por (HernándezSilva \& Flores, 2017), en su estudio el Aula invertida, mediada por el uso de plataformas virtuales se identificó ventajas y desventajas mencionadas por los estudiantes que participaron en la investigación, al terminar el semestre, volviendo ahora a los aspectos positivos tenemos: el desarrollo de hábitos y autorregulación para el aprendizaje, en cuanto a los aspectos negativos supone la dificultad constate de revisar con anticipación antes de iniciar la clase en plataformas virtuales como parte de los prerrequisitos, otro aspecto negativo es creer que el aula invertida asegura éxito en el proceso de aprendizaje, es básico articular sistemáticamente el ambiente de aprendizaje, cabe también indicar que si el ambiente en donde se desarrolla el proceso sea favorable será preciso ajustar las estrategias didácticas, responsabilidad que recae puntualmente en el docente. 


\section{METODOLOGÍA}

La metodología es de tipo descriptiva correlacional con cálculo de Chi-cuadrado de Pearson, mediante un diseño de campo no experimental. La población a la que estuvo orientada a estudiantes y padres de familia o representantes legales del tercer año de Educación General Básica paralelo "A" de la Unidad Educativa Fiscomisional "Mariana de Jesús" del cantón el tambo Provincia del Cañar - Ecuador.

Se aplicó la técnica de la encuesta, la cual fue dirigida a representantes legales, o padres de familia del tercero "A", mediante un instrumento que contó con diez preguntas cerradas, y validado con el Alpha de Cronbach, cuyo valor fue 0.745 , se espera obtener un epítome de opiniones sobre cómo la aplicación del aula invertida puede aportar al aprendizaje de estudiantes, desde la percepción de los 30 representantes o padres de familia Para identificar cuáles son los aspectos positivos y los aspectos negativos que se han evidenciado a través del uso de esta metodología, los datos obtenidos fueron procesados desde la estadística descriptiva y correlacional.

\section{RESULTADOS}

Al realizar el análisis de normalidad de las 8 variables se determinó que todas las variables son paramétricas, El estudio de los resultados sigue lo establecido en la metodologia mediante un análisis cuantitativo no experimental. 


\section{Tabla 1.}

A mejorado el aprendizaje con la Flipped Classroom.

\begin{tabular}{llllll}
\hline & & Frecuencia & Porcentaje & $\begin{array}{l}\text { Porcentaje } \\
\text { válido }\end{array}$ & $\begin{array}{l}\text { Porcentaje } \\
\text { acumulado }\end{array}$ \\
\hline Válidos & Siempre & 11 & 36,7 & 36,7 & 36,7 \\
& Casi siempre & 12 & 40,0 & 40,0 & 76,7 \\
& Frecuentemente & 5 & 16,7 & 16,7 & 93,3 \\
& A veces & 2 & 6,7 & 6,7 & 100,0 \\
\cline { 3 - 5 } & Total & 100,0 & 100,0 & \\
\hline
\end{tabular}

Fuente: Encuesta.

La tabla 1. evidencia que el 93,3 por ciento de los Padres de familia o Representantes legales afirman que con la utilización de esta metodología activa el aprendizaje de sus hijos a mejorado significativamente, razón por la que la motivación que la flipped classroom genera en los estudiantes es alentadora, para proponer que los docentes utilicen dentro de sus metodologías el aula invertida en el proceso de enseñanza.

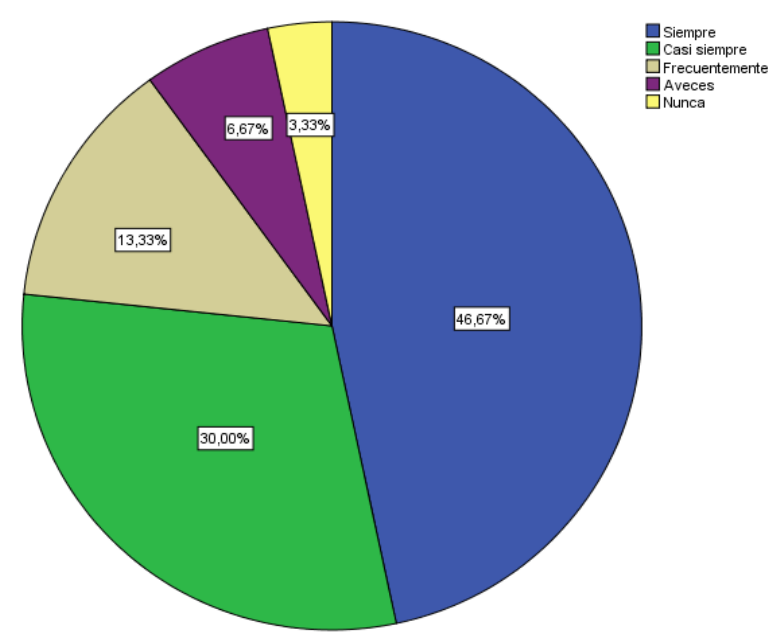

Figura 1. Recomienda a los docentes utilizar la metodología de aula invertida.

Fuente: Encuesta. 
La figura 1 evidencia que $46,67 \%$ de los padres de familia recomiendan a los profesores que siempre deben utilizar la metodología activa flipped classroom en el proceso de enseñanza aprendizaje con los estudiantes, frente a un 30\% que prácticamente coinciden que la metodología de debe utilizar casi siempre arrojando así un resultado positivo a favor de la incidencia que tiene la metodologia dentro del proceso educativo. Esto desde el punto de vuista de los padres de familia o representantes legales.

En la tabla 2 se realizó un análisis Descriptivo mediante la relación de Chicuadrado en donde se demuestra una relación entre las variables puesto que la sig bilateral tiene un valor de 0,000 inferiror a 0.05 asumiendo dicha relación entre las mismas. Pues se evidencia notablemente la eficacia de la metodologia, siempre que el material sea revisado de forma oportuna por el estudiante, como resultado de aquello se obtiene una mayor motivación en las clases. Es por esto que al momento de implementar esta clase de metodologías en el aula la revicion de materiales es parte primordial a tener en cuenta.

\section{Tabla 2.}

Relación entre El aula invertida motiva al estudiante y a mejorado el aprendizaje con Flipped Classroom.

\begin{tabular}{|c|c|c|c|c|c|c|}
\hline & \multicolumn{4}{|c|}{ A mejorado el aprendizaje con Flipped Classroom } & \multirow[b]{2}{*}{ Total } \\
\hline & & A veces & Frecuentemente & $\begin{array}{l}\text { Casi } \\
\text { siempre }\end{array}$ & Siempre & \\
\hline \multirow{4}{*}{$\begin{array}{l}\text { El aula invertida motiva } \\
\text { al estudiante }\end{array}$} & A veces & 2 & 0 & 0 & 0 & 2 \\
\hline & Frecuentemente & 0 & 1 & 3 & 0 & 4 \\
\hline & Casi siempre & 0 & 2 & 4 & 1 & 7 \\
\hline & Siempre & 0 & 2 & 5 & 10 & 17 \\
\hline \multicolumn{2}{|l|}{ Total } & 2 & 5 & 12 & 11 & 30 \\
\hline \multicolumn{7}{|c|}{ Pruebas de chi-cuadrado } \\
\hline \multirow{2}{*}{\multicolumn{2}{|c|}{ Chi-cuadrado de Pearson }} & \multicolumn{2}{|c|}{ Valor $\quad$ gl } & \multicolumn{3}{|c|}{ Sig. asintótica (bilateral) } \\
\hline & ion & $37,788^{a}$ & 9 & \multicolumn{2}{|c|}{0,000} & \\
\hline \multicolumn{2}{|c|}{ Razón de verosimilitudes } & 23,524 & 9 & \multicolumn{2}{|c|}{0,005} & \\
\hline \multicolumn{2}{|c|}{ Asociación lineal por lineal } & 12,426 & 1 & \multicolumn{2}{|c|}{0,000} & \\
\hline \multicolumn{2}{|c|}{$\mathrm{N}$ de casos válidos } & \multicolumn{2}{|l|}{30} & & & \\
\hline
\end{tabular}

Fuente: Encuesta. 


\section{PROPUESTA}

\section{Taller de capacitación a la comunidad educativa sobre el Aula Invertida}

El taller de capacitación a la comunidad educativa se realizó con un enfoque en 4 aspectos definidos en las siguientes fases: planificar, ejecutar, controlar y actuar con el propósito de mejorar el desempeño estudiantil de la institución Unidad Educativa Mariana de Jesús del Cantón El Tambo.

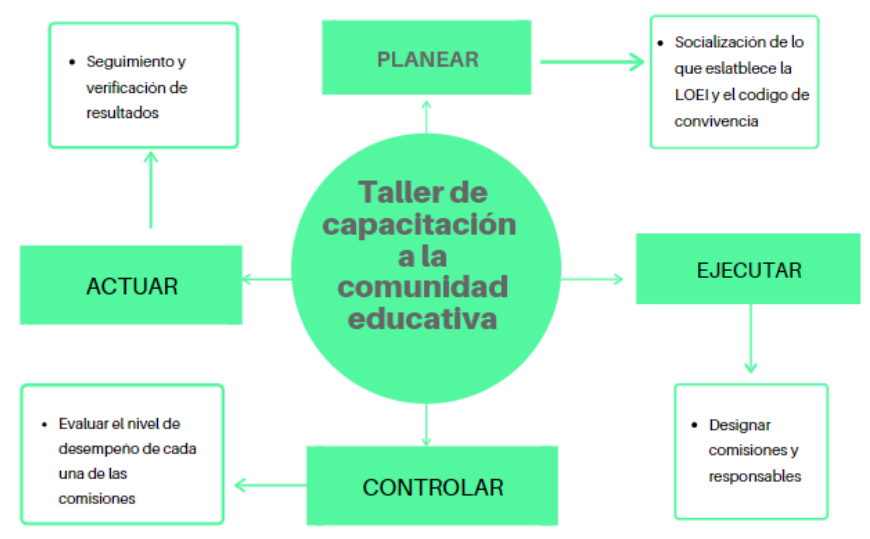

Figura 2. Esquema de la propuesta del taller de capacitación a la comunidad educativa sobre el aula invertida.

Fuente: Los autores.

Se detallan las fases de ejecución de la propuesta:

Planificar: Se ha partido desde la Ley Orgánica de Educación Intercultural [LOEI] en donde se establece que los docentes tendrán que mantenerse en constantes capacitaciones y parte fundamental de esto es el uso de nuevas tecnologías, así mismo insta a los representantes a ser parte activa en el proceso de aprendizaje de sus hijos, de igual manera parte del perfil de salida de los estudiantes es la innovación, Adicional a esto se apoya en el código de convivencia institucional y al que toda la comunidad 
educativa debe cumplir. Se establecerá un compromiso escrito por parte de los representantes, profesores, directivos y estudiantes Los objetivos del modelo propuesto, se plantea estrategias para dar solución a la problemática del bajo rendimiento académico mediante la instauración en nuestra institución de metodologías activas por parte de los docentes, previa capacitación a padres de familia y a estudiantes sobre el accionar de la metodología.

Ejecutar: Tiene como fin designar responsabilidades a cada miembro de la comunidad educativa mediante comisiones para representantes o padres de familia, estudiantes, directivos y personal docente, mismas que trabajaran de forma conjunta con cada grupo designado para facilitar el desarrollo programado de los talleres. Adicional a esto se designarán una comisión de protocolo misma que velara para tener los recursos físicos, instalaciones adecuadas, elaborar horarios para los talleres de capacitación y llevara un registro de asistencia en la que se evidencie la participación de cada miembro de la institución.

Controlar: Se medirá el nivel de desempeño de las comisiones en los distintos procesos se propone el uso de indicadores de desempeño, los cuales permitirán identificar problemas que pudieran surgir a lo largo del tiempo que dure el taller, para de esta manera poder re-planificar las temáticas abordadas, Establecer reglas, horarios de trabajo, situaciones de permisos, e inasistencias.

Actuar: Está enfocado a una fase de seguimiento y verificación del desarrollo, avances y resultados del taller de capacitación a la comunidad educativa sobre el aula invertida. que estará a cargo de los directivos y consejo ejecutivo de la institución para medir el alcance del objetivo planteado del taller. Es así como se prevee preparar a los docentes, padres de familia, representantes legales y estudiantes sobre el accionar que ha de llevarse a cabo al implementar esta metodología activa Flipped Classroom. 


\section{CONCLUSIONES}

En base al trabajo realizado en la investigación se concluye que la metodología Flipped Classroom o aula invertida goza de la aceptación del 46,67\% de los padres de familia quienes expresan que mediante esta metodolgía han evidenciado un cambio positivo en cuanto al redimiento de sus hijos o de sus representados por lo que recomiendan a los profesores que utilicen la metodología activa flipped classroom en el proceso de enseñanza aprendizaje con los estudiantes.

Consecuentemente un $30 \%$ de encuestados coinciden que la metodología utilizada debe estar presente dentro de la amplia gama de metodologías utilizadas por los maestros, evidenciandose así la actual popularidad de las metodologías activas en si del aula invertida ,se evidencia el eminente cambio al que estamos sujetos la comunidad educativa.

En resumen es un llamado a abandonar la educación tradicional que si bien en su momento aportó positivamente en la educación ahora se encuentra en deshuso ,hoy el entorno inmediato de los estudiantes, representantes, directivos,padres de familia,y docentes es diferente pues nos encontramos frente a nuevas tecnologías que permiten un mejor desempeño y abren las puertas a nuevas posibilidades para acceder al conocimiento.

De lo que se concluye que la Flipped Classroom o aula invertida es de agrado de estudiantes y padres de familia que han vivido la experiencia de comparar el rendimiento académico con esta metodología activa, que en sí misma ofrece la oportunidad al estudiante de ser parte activa de su aprendizaje y no limita sus posibilidades de aprender únicamente a lo propuesto por el docente o profesor sino por el contario estimula la investigación a través del uso de la tecnología.

Los estudiantes al utilizar un amplio y variado abanico de recursos y herramientas tecnológicas con las que se encuentra familiarizado, genera una seguridad y motivación esto en comparación con la metodología tradicional en la cual el papel protagónico lo tiene el docente y el estudiante se rige únicamente a lo que el docente pueda aportar a 
su conocimiento, no es raro encontrarse también con estudiantes rezagados, con escasa o nula motivación a estudiar y en parte considero que esto surge a partir del uso de recursos caducos en las clases, los mismos que no estimulan la participación estudiantil menos la investigación y son recursos que los estudiantes no manipulan o no manejan en su entorno inmediato.

En síntesis, hay que tener presente que solamente con la implementación de metodologías activas y en sí de la Flipped Classroom no todo está resuelto, pues hay que considerar situaciones como el no contar con los recursos tecnológicos, o económicos que satisfagan las necesidades que implica esta metodología.

Otro factor relevante dentro del análisis es considerar el grado de responsabilidad en los educandos para revisar el material que el profesor envíe con anterioridad para evitar posibles rezagos al momento de aprender.

Se desprende como una gran ventaja que los estudiantes dispongan del material para mediante estos recursos ellos lo puedan adaptar a su individualidad estudiantil hasta lograr su aprendizaje de manera personalizada.

Otro punto considerar es el acceso a recursos tecnológicos propios del estudiante y no de sus padres o hermanos que limitarían el aprendizaje a cuando puedan tener acceso a estos dispositivos, Pues la situación económica producto de la pandemia no es la mejor. Otra conclusión a tener en cuenta es que el tiempo de los videos Flipped, lecturas, pódcast o cualquier otro tipo de material utilizado deben ser breves y precisos que faciliten la comprensión.

\section{FINANCIAMIENTO}

No monetario.

\section{AGRADECIMIENTO}

A la Corporación Eléctrica del Ecuador y la Jefatura de Posgrados de la Universidad Católica de Cuenca por permitir el desarrollo y fomento de la investigación. 


\section{REFERENCIAS CONSULTADAS}

Aguayo-Vergara, M., Bravo Molina, M., Nocetti de la Barra, A., Concha Sarabia, L., \& Aburto Godoy, R. (2018). Perspectiva estudiantil del modelo pedagógico flipped classroom o aula invertida en el aprendizaje del Inglés como lengua extranjera [Student perspective of the pedagogical model flipped classroom or inverted classroom in learning English as a foreign lan. Revista Educación, 43, 97-112. https://doi.org/10.15517/revedu.v43i1.31529

Belfar, A., Defrisbourg, F., \& Nowak, T. (2003). Crises asystoliques syncopes convulsivantes. Epilepsies, 15(2), 99-100.

Domínguez, L. C., Vega, N. V., Espitia, E. L., Sanabria, Álvaro E., Corso, C., Serna, A. M., \& Osorio, C. (2015). Impacto de la estrategia de aula invertida en el ambiente de aprendizaje en cirugía: una comparación con la clase magistral [Impact of the flipped classroom strategy on the learning environment in surgery: a comparison with the master class]. Biomédica, 35(4), 513-21. https://doi.org/10.7705/biomedica.v35i4.2640

Granados-López, H., \& García-Zuluaga, C. L. (2016). El modelo de aprendizaje experiencial como alternativa para mejorar el proceso de aprendizaje en el aula [The Experiential Learning Model as an Alternative for Improving the Learning Process in the Classroom]. Ánfora, 23(41), 37-54. https://doi.org/10.30854/anf.v23.n41.2016.140

Hernández-Silva, C., \& Flores, S. T. (2017). Inverted classroom mediated by the use of virtual platforms: A case study in the training of physics teachers. Estudios Pedagogicos, 43(3), 193-204. $\quad$ https://doi.org/10.4067/S0718$\underline{07052017000300011}$

Jensen, J. L., Kummer, T. A., \& d M Godoy, P. D. (2015). Improvements from a flipped classroom may simply be the fruits of active learning. CBE life sciences education, 14(1), ar5. https://doi.org/10.1187/cbe.14-08-0129

Mendoza-Castillo, L. (2020). Lo que la pandemia nos enseñó sobre la educación a distancia [What the pandemic taught us about distance education]. Revista Latinoamericana De Estudios Educativos, 50(ESPECIAL), 343-352. https://doi.org/10.48102/rlee.2020.50.ESPECIAL.119 
Molina-Naranjo, J. M., Lavandero García, J., \& Hernández Rabell, L. M. (2018). El modelo educativo como fundamento del accionar universitario.: Experiencia de la Universidad Técnica de Manabí, Ecuador [The educational model as the foundation of university action. : Experience of the Technical University of Manabí, Ecuador]. Revista Cubana de Educación Superior, 37(2), 151-164.

Parra-Acosta, H., Tobón, S., \& López Loya, J. (2015). Socioformative teaching and academic performance in higher education [Enseñanza socioformativa y rendimiento académico en la educación superior]. Paradígma, 36(1), 42-55.

Sánchez-Cruzado, C. (2017). Flipped classroom. La clase invertida, una realidad en la Facultad de Ciencias de la Educación de la Universidad de Málaga [The inverted class, a reality at the Faculty of Education of the University of Malaga]. https://hdl.handle.net/10630/14993

Sosa-Díaz, M. J., \& Palau-Martín, R. F. (2018). Flipped Classroom para la Formación del Profesorado: Perspectiva del alumnado [Flipped Classroom for Teacher Education: Student Perspective]. REDU. Revista de Docencia Universitaria, 16(2), 249-264. https://doi.org/10.4995/redu.2018.7911 